\title{
UNIVALENT FUNCTIONS AND NONANALYTIC CURVES
}

\author{
A. W. GOODMAN
}

The object of this note is to give parametric equations of a very elementary form for a simple closed convex curve with a continuously turning tangent, with no analytic subarc. The work is based on the following result.

THEOREM 1. Let

$$
f(z)=z+\sum_{n=2}^{\infty} a_{n} z^{n}, \quad \text { for }|z|<1 .
$$

If

$$
\sum_{n=2}^{\infty} n\left|a_{n}\right| \leqq 1
$$

then $f(z)$ is univalent in $|z|<1$ and maps that region onto a region that is starlike with respect to the origin. If

$$
\sum_{n=2}^{\infty} n^{2}\left|a_{n}\right| \leqq 1
$$

then $f(z)$ is univalent in $|z|<1$, and maps that region onto a convex region.

The two examples $z+(1+\epsilon) z^{2} / 2$ and $z+(1+\epsilon) z^{2} / 4$ with $\epsilon>0$, show that the right sides of (2) and (3) cannot be increased without destroying respectively the starlikeness and convexity of the image regions.

This theorem was first discovered by Alexander [1], but according to Remak [6], Alexander's proof contains an error. Remak supplied a correct proof, and we give here a second proof of this theorem which is somewhat different and yields a little more information about the mapping function, than the one given by Remak. The condition (2) on the power series has been investigated further by Yan'-Sin Čin [2], Mullender [3; 4], and Schild [7].

It is well known that the condition

$$
\Re \frac{z f^{\prime}(z)}{f(z)}>0
$$

for $|z|<1$,

is both necessary and sufficient that $f(z)$ given by (1) map $|z|<1$

Received by the editors August 27, 1956. 
onto a region that is starlike with respect to the origin [5, p. 221]. Further, if

$$
F(z)=z+\sum_{n=2}^{\infty} b_{n} z^{n}
$$

maps $|z|<1$, onto a convex region then $f(z)=z F^{\prime}(z)$ maps $|z|<1$ onto a region starlike with respect to the origin, and conversely $[5$, p. 223]. Now the condition (2) for $f(z)$ is equivalent to the condition (3) for $F(z)$, so that it suffices to prove only the first part of the theorem.

Suppose now that the inequality (2) is valid for $f(z)$ and write

Then

$$
\begin{aligned}
\frac{z f^{\prime}(z)}{f(z)} & =\frac{1+2 a_{2} z+3 a_{3} z^{2}+\cdots+n a_{n} z^{n-1}+\cdots}{1+a_{2} z+a_{3} z^{2}+\cdots+a_{n} z^{n-1}+\cdots} \\
& =1+\sum_{n=1}^{\infty} B_{n} z^{n} .
\end{aligned}
$$

$$
\begin{aligned}
& B_{1}=a_{2}, \\
& B_{2}=2 a_{3}+a_{2} B_{1}, \\
& B_{3}=3 a_{4}+a_{2} B_{2}+a_{3} B_{1}, \\
& \dot{B_{n-1}}=(n-1) a_{n}+a_{2} B_{n-2}+a_{3} B_{n-3}+\cdots+a_{n-1} B_{1},
\end{aligned}
$$

and hence for $n \geqq 2$

$$
\sum_{k=1}^{n-1} B_{k}=\sum_{k=2}^{n}(k-1) a_{k}+a_{2} \sum_{k=1}^{n-2} B_{k}+a_{3} \sum_{k=1}^{n-3} B_{k}+\cdots+a_{n-1} B_{1} .
$$

Now the inequality (2) implies that $\left|B_{1}\right|=\left|a_{2}\right| \leqq 1 / 2$, and if we assume that $\left|\sum_{k=1}^{s} B_{k}\right| \leqq 1$ for $s=1,2, \cdots, n-2$, then the equation (7) yields

$$
\begin{aligned}
\left|\sum_{k=1}^{n-1} B_{k}\right| & \leqq \sum_{k=2}^{n}(k-1)\left|a_{k}\right|+\left|a_{2}\right|+\left|a_{3}\right|+\cdots+\left|a_{n-1}\right| \\
& \leqq \sum_{k=2}^{n} k\left|a_{k}\right| \leqq 1,
\end{aligned}
$$

again using the inequality (2). Thus by induction the inequality ( 8 ) is valid for each integer $n \geqq 2$.

It now follows from Abel's lemma $[8, \text { p. 6 }]^{1}$ that for $0<r \leqq 1$,

1 The statement and proof of Abel's lemma given by Titchmarsh require that $B_{h}$ is real; however, it is easily generalized to complex series. 
$\left|B_{1} r+B_{2} r^{2}+\cdots B_{n} r^{n}\right| \leqq 1$. Therefore for $z=r$, we have from equation (6)

$$
\left|\frac{z f^{\prime}(z)}{f(z)}-1\right| \leqq 1 .
$$

But the inequality (2) is invariant under rotations of the $z$ and $w$ planes, and the starlike character of the image domain is likewise invariant, under these rotations, so that any $z$ in the unit circle may, by a preliminary rotation be placed in the interval $[0,1]$. Thus the inequality (2) implies that the inequality (9) is valid throughout the unit circle. The case of the equality sign in (9) for an interior point of the unit circle, can easily be rejected on the basis of the normalization $f^{\prime}(0)=1$. But the inequality (9) implies the inequality (4), and the proof of the theorem is complete.

For a specific example, set

$$
F(z)=z+\sum_{k=1}^{\infty} \frac{z^{2 k}}{16^{k}} .
$$

By the Hadamard gap theorem [8, p. 223], the circle $|z|=1$ is a natural boundary for $F(z)$. But the series for $F^{\prime}(z)$ is absolutely and uniformly convergent on $|z|=1$, so that the image curve $\Gamma$, of $|z|=1$ under $F(z)$, has a continuously turning tangent. Further, the inequality (3) is satisfied, since for $F(z)$

$$
\sum_{n=2}^{\infty} n^{2}\left|a_{n}\right|=\sum_{k=1}^{\infty}\left(2^{k}\right)^{2} \frac{1}{16^{k}}=\sum_{k=1}^{\infty}\left(\frac{1}{4}\right)^{k}=\frac{1}{3}<1,
$$

so that $F(z)$ is univalent in $|z|<1$, and maps that region onto a convex region, whose boundary is $\Gamma$. Therefore $\Gamma$ is a simple closed convex curve, with the parametric equations

$$
\begin{aligned}
& u=\sum_{k=0}^{\infty} \frac{1}{16^{k}} \cos 2^{k} \theta, \\
& v=\sum_{k=0}^{\infty} \frac{1}{16^{k}} \sin 2^{k} \theta .
\end{aligned}
$$

Finally, if any arc of $\Gamma$ were analytic, so that $u$ and $v$ could be expressed by convergent power series in a real variable $t$, with real coefficients, then the function $G(t)=u(t)+i v(t)$ would be analytic in a circle containing a segment of the real axis. Such a function would provide for the continuation of $F(z)$ across the boundary of $|z|=1$ 
in an obvious way. But this is impossible, since $|z|=1$ is already a natural boundary for $F(z)$.

\section{REFERENCES}

1. J. W. Alexander, Functions which map the interior of the unit circle upon simple regions, Ann. of Math. vol. 17 (1915) pp. 12-22.

2. Yan'-Sin Čin, On the arguments of the coefficients of the expansion of a univalent function, Acta Math. Sinica vol. 4 (1954) pp. 81-86 (In Chinese, Russian summary).

3. P. Mullender, On some conformal mappings, Simon Stevin, vol. 26 (1949) pp. 136-142 (in Dutch).

4. - On some conformal mappings, Simon Stevin vol. 30 (1954) pp. 44-47 (in Dutch).

5. Z. Nehari, Conformal mapping, McGraw-Hill, 1952.

6. R. Remak, Ueber eine spezielle Klasse schlichter konformer Abbildungen des Einheitskreises, Mathematica B vol. 11 (1943) pp. 175-192.

7. A. Schild, On a class of functions schlicht in the unit circle, Proc. Amer. Math. Soc. vol. 5 (1954) pp. 115-120.

8. E. C. Titchmarsh, The theory of functions, Oxford University Press, 1932.

The Institute for Advanced Study ANd

THE UNIVERSITY OF KeNTUCKY 